\title{
Combining Eye Tracking and Conventional Techniques for Indications of User-Adaptability
}

\author{
Ekaterini Tzanidou ${ }^{1}$, Marian Petre ${ }^{1}$, Shailey Minocha ${ }^{1}$, and Andrew Grayson ${ }^{2}$ \\ ${ }^{1}$ Department of Computing, The Open University, Walton Hall, \\ Milton Keynes MK7 6AA, UK \\ \{e.tzanidou, m.petre, s.minocha\} @open.ac.uk \\ ${ }^{2}$ Division of Psychology, Nottingham Trent University, Burton Street, \\ Nottingham, NG1 4BU, UK \\ andy.grayson@ntu.ac.uk
}

\begin{abstract}
We have captured and analysed users' eye movements by means of an eye-tracking device to re-visit existing web design guidelines. The study reported here examines how quickly users adapt to an unfamiliar design layout and, in particular, how quickly they adjust their expectations of where to look for a given target link during repeated exposures to a new layout. Eye movement-based metrics such as time to target fixation, location of first fixation and scan path (sequence of fixations) were applied to capture users' eye movements. These metrics were then applied to analyse the effects of repeated exposures and of design layouts of websites. More exposures led to decreased time to target fixation, indicating that user-adaptation occurred. The visual characteristics of the target link also influenced visual search behaviour. Qualitative complementary data such as the users' frequency and purpose of internet usage, users' expectations about the target link added value to the eyemovement data.
\end{abstract}

\section{Introduction}

As Jones and Dumais [21] stated it "it is not enough to know what we are looking for, we must also know where to look for it" (pg.43). This is evident when users encounter web pages where they are often presented with an overwhelming amount of information, with a mix of visual and textual design elements clamouring for attention. Thus, understanding the factors that influence user's visual search behaviour on the user interface is important so as to design usable user interfaces.

Existing design guidelines embody assumptions that visual search behaviour is shaped by expectations, hence they suggest designing user interfaces that conform to conventions. Nielsen et al. [26] underlines the importance of maintaining consistency with other websites and pages. "All web pages are much the same from the user's perspective, they share interaction techniques, they are downloaded (slowly) from the internet, and they have relatively similar layouts. Those similarities are in fact good because they allow users a measure of transfer of skill from one site to the next. Users complain bitterly when a site doesn't try to use navigation from the majority of other sites" (pg. 189). But how do users learn conventions and develop expectations? Ehret 
[5] suggests that when locations of design elements remain constant, performance improves over exposures as users learn placements of design elements and focus/limit the scope of their visual search behaviour. The consistent placement of design elements influences visual search.

But a recent eye tracking study by McCarthy et al. [25] investigated the impact of changing the placement of design elements and how users performed when viewing the element in unexpected placements. The authors found that following conventions with other websites did not matter, as users quickly adapted to design layouts with unexpected placement of design elements.

So is it important to follow consistency with other websites or is it acceptable to place design elements in non-consistent locations on the user interface? The study reported in this paper used an eye tracking device to capture users' eye movements and investigated how quickly users adapt to different placements of a design element over repeated exposures. Specifically, the study focused on one design element, the 'About Us' link on a website, and addressed the following questions:

Q1: Do users adapt their expectations of where to look for when presented with repeated exposures of the 'About Us' link in a consistent, but unconventional, position?

Q2: Do users adapt quickly to alternative design layouts such as finding the 'About Us' link in an unconventional position?

Q3: Where do users look first, after the repeated exposures, on pages that do not contain any 'About Us' link?

Previous research as conducted by McCarthy et al. [25] had investigated whether placing design elements, such as menu items, in unexpected positions has an impact on visual search behaviour in terms of search performance. Variations of simple and complex web pages presented menu items in 3 different locations (top, left, right) for 3 different tasks. Each type of web page received three page visits. We have applied a different experiment design: consistent placement of a design element over repeated exposures but placed the design element at an unconventional position (that is, not as per the guidelines) to explore user adaptation. Moreover users were also presented with web pages that didn't contain the 'target' design element (About Us link) in order to explore users' expectations of its location. Complementing eye movement data and conventional techniques, such as self-reports of expectations and preferences as elicited from pre- and post-session questions, has enabled a better understanding and interpretation of the eye-movement data. User-adaptation occurred over repeated exposures of the web pages and a change in user's expectations was detected by where the users looked for the target link on web pages on which the target link was absent.

\subsection{Visual Search Behaviour}

Theories of visual search, as reported by Horowitz and Wolfe [17], conclude that visual search relies on accumulating information about the identity of design elements over time. This knowledge enables designers to structure the user interface effectively and influence the user's visual search behaviour. Post-cognitive modelling research, as cited in [17], has demonstrated that people use anticipatory location information to 
guide visual search, and that visual features sometimes guide the visual search (i.e., expectations and salience) [18]. It is through visual search primarily that users locate the content and control for their web-based tasks. Despite extensive research into visual search behaviour in disciplines such as psychology, recent research in HCI [15, 18] has underlined the importance of developing a unified understanding of the visual search in HCI. Visual search behaviour on websites is influenced by expectations about what is being looked for and where it might be located. Pirolli and Card [30] talks about the design layout of the display as a bottom-up influence and expectations as a top-down influence. Bottom-up processing refers to the design elements influencing the visual scene itself, such as presentation format, colour, position, whereas top-down processing refers to the expectations the users develop such as the cognitive processes when viewing a scene. The interactions between top-down and bottom-up influences is identified as Information Scent or Information Foraging [30]. Unless the design elements such as colour, menu items, graphs (bottom-up) are looked at, there is no 'scent', and therefore there is no basis for selection.

\section{Eye Tracking to Assess Usability}

Conventional usability evaluation techniques such as user-observations, think-aloud protocols, questionnaires and interviews focus more on the activities of user performance rather than understanding the cognitive processes of the users [12]. Therefore, aspects of task performance such as ease of navigation, efficiency of selecting the 'right' menu item, or time taken to complete a task can be captured but the inferences of cognitive processes are more difficult to explain. Eye tracking studies in cognitive psychology have established that eye movements give an insight into the users' cognitive processes (e.g. [23]). Eye movements in reading and information processing have been studied by Rayner and Pollatsek [31] and they concluded that eye movements indicate how easy or difficult it is to process a display. The use of eye tracking in HCI is not a new concept, as Jacob and Karn [20] have illustrated in their recent review of eye tracking studies. In 1954, Fitts [7] was the first to conduct a systematic eye tracking study of pilots using cockpit controls and instruments. In recent years eye tracking devices have become more affordable, and the technology has improved, enabling an increasing number of HCI researchers to engage in eye tracking studies [4]. In general eye tracking data is used to support recommendations for how a user interface should be designed for usability, rather than provide a broad assessment of the interface's overall usability [12].

Previous research [3, 9, 10, 32] has established that specific design elements influence eye movements in a predictable way, and they demonstrate that eye tracking metrics are sensitive enough to detect them. The authors have compared eye tracking data with performance times, completion rates, and accuracy of task responses. For example, Goldberg and Kotval [10] manipulated items' grouping labelling on the tool bars of several versions of a simulated drawing package which were shown to interface designers for their (subjective) usability ratings. The eye tracking data as captured from real users was then correlated with the designers' subjective ratings. Eye tracking metrics such as duration of fixations, saccadic amplitude and fixation/saccade ratio were not found to be useful in predicting the differences in 
usability ratings. Metrics such as scan path length, number of fixations, and number of saccades were found to be more sensitive in highlighting the differences in the usability ratings.

The effectiveness of eye movement-based metrics has been explored to suggest ways of applying them in order to answer specific research questions regarding visual search behaviour. For example, Fitts et al. [8] distinguish between frequency and duration of fixations with duration reflecting difficulty of information extraction and frequency reflecting the importance of that area of the interface.

Eye tracking has been applied in HCI in two ways: as a real-time input device and its use as a usability evaluation technique [20]. In the study reported in this paper, eye tracking has been applied for usability evaluation of websites.

\subsection{Eye Tracking Studies of Websites}

Granka et al. [13] have reported that only a small number of studies have been conducted on eye movement behaviour on web pages. Visual preferences of text and images have been explored by two studies [6, 24]. Ellis et al. [6] demonstrated that users completed tasks more quickly and easily on text-based screens, although they preferred image-based screens. The Stanford Poynter Project study [24] examined how users read on-line and off-line news; they found that text was viewed more than images for readers who read on-line news, whereas the opposite occurred for readers who read off-line news.

Two studies [11, 22] have examined the navigational styles on user interfaces of web pages. Josephson and Holmes [22] examined users' scan paths on different kinds of images widely used on the internet to test Norton and Stark's scan path theory and identified strong similarities among scan paths, suggesting that different users' eye movements may follow a 'habitually preferred path'. Goldberg et al. [11] captured navigational styles of users freely navigating web portal pages. They concluded that headers of links are not always viewed before the main body. This research went on to develop specific design recommendations for portal pages and suggest design changes based on eye movement data.

The studies discussed above explored navigational styles that users apply on both offline and online channels but these studies did not investigate the factors that influence the navigation styles. More recent studies [e.g. 27, 29] have aimed to understand the factors that influence visual search behaviour. Pan et al. [29] investigated some of these factors, such as individual differences, design characteristics of the web pages, the order in which web pages are viewed and different tasks that were given to the users to complete. Gender and viewing order were found to be the key determinants of visual search behaviour. Men applied different scan paths from women and the order in which the stimuli were presented influenced the scan paths as well.

The Stanford Poynter Project [27] extended their previous work [24] on how users read news websites. They applied a more methodological approach in their latter study. Some of the key points of their latest study [27] suggest that users navigate more on the upper part of news websites rather than left or right of the page. The size of text was found to be influential in terms of encouraging focused viewing 
behaviour; smaller text drew more fixations while larger sizes promoted lighter scanning. The users fixated more on headlines with large text rather than headlines with small text.

\subsection{Eye Movement-Based Metrics and Terminology}

As there is no standard way of defining a single fixation operationally, and there are different psychological theories about the relationship between eye movements and cognitive processes [16]; there is a need to ensure a common understanding of the use of terms and definitions related to eye-tracking in the context they are used. The following eye movement-based metrics were applied in our study being reported here: i) Time to target fixation: the time users need to fixate on the target link gives a basis of performance measurement when a specific search target exists. Since we are interested in investigating how quickly or slowly the target link is fixated, the time to target fixation is an indication of user performance. The target link in our study is the 'About Us' link; ii) Location of fixation: the location of fixations is used as an indicator of where users look on a web page to locate the target link; iii) Initial gaze: initial gaze measures the user's first gaze during the first 50 milliseconds of the web page appearing on the screen in order to examine where users expect to find the target link; iv) Entry point: entry point measures the user's first fixation within the first 250 milliseconds of the web page appearing on the screen in order to examine which design elements first draw user's visual attention; v) Scan path: the sequence of fixations indicates the order in which the user looked at areas on the web page.

\section{Method}

The eye tracking study reported here collected four types of data: i) A background questionnaire to elicit the participants' internet experience and typical frequency of usage; ii) Pre-session questions to elicit expectations about the target link (About Us link); iii) Eye tracking data to measure visual search behaviour; and iv) Post-session questions to address perceptions and preferences about the About Us link.

\subsection{Experiment Design}

A counterbalanced experiment design [2] was applied varying the ten exposures of web pages to eliminate possible order effects. Ten web pages of E-Commerce sites were selected and amended so that they would appear in each of the three different exposure styles. So, for example, each web page was amended in order to have: i) The 'About Us' link at the bottom of the page, ii) The 'About Us' link at the top of the page, and iii) No 'About Us' link. The description of each exposure and purpose is presented in Table 1.

The study had two sets of hypotheses: The first set addressed the effect of the consistent placement of the target link (About Us link) for exposures 2 to 7 and predicted that the placement of the target link at the bottom of the page over six repeated exposures would result in the participants' decrease in the time to target fixation and also change in participant's expectations of where to find the target link. 
The second set examined the effect of the alternative placement of the target link (About Us link) and predicted that the placement of the target link at the top of the page in Exposure 8 would result in quick adaptation to an unexpected design layout. Specifically we anticipated that the two first sets of hypotheses will be evidenced by shorter times to target fixation, modifications of scan patterns, change of location of first fixations and self-report of preferences and expectations.

Table 1. Description and purpose of exposures

\begin{tabular}{|c|l|l|}
\hline Exposures & \multicolumn{1}{|c|}{ Description } & \multicolumn{1}{c|}{ Purpose } \\
\hline Exposure 1 & $\begin{array}{l}\text { Did not include the 'About } \\
\text { Us' link }\end{array}$ & $\begin{array}{l}\text { To explore users' expectations of where to } \\
\text { find the 'About Us' link before the repeated } \\
\text { exposure session }\end{array}$ \\
\hline $\begin{array}{c}\text { Exposure } \\
2-7\end{array}$ & $\begin{array}{l}\text { Six repeated exposures } \\
\text { where the 'About Us' link } \\
\text { appeared at the bottom of } \\
\text { the page }\end{array}$ & $\begin{array}{l}\text { To examine the effect of consistent design } \\
\text { element placement on visual search } \\
\text { behaviour. }\end{array}$ \\
\hline Exposure 8 & $\begin{array}{l}\text { The 'About Us' link } \\
\text { appeared at the top of the } \\
\text { page }\end{array}$ & $\begin{array}{l}\text { To capture the users' visual reactions when } \\
\text { introduced to an alternative design layout } \\
\text { after being presented with the repeated } \\
\text { exposures in which the 'About Us' link } \\
\text { appeared at the bottom of the page }\end{array}$ \\
\hline Exposure 9 & $\begin{array}{l}\text { The 'About Us' link } \\
\text { appeared at the bottom of } \\
\text { the page again }\end{array}$ & $\begin{array}{l}\text { To assess persistence of any affect of } \\
\text { repeated exposures on visual search } \\
\text { behaviour }\end{array}$ \\
\hline Exposure 10 & $\begin{array}{l}\text { Did not include the 'About } \\
\text { Us' link }\end{array}$ & $\begin{array}{l}\text { To explore users' expectations where to find } \\
\text { the 'About Us' link after the repeated } \\
\text { exposure session }\end{array}$ \\
\hline
\end{tabular}

\subsection{Participants}

Ten volunteers (5 male and 5 female) with age range of 22-56 from the staff and postgraduate student population of the Open University participated in this study. Five participants were regular (using the internet 2-3 times per day) internet users and five participants were frequent (using the internet throughout the day as part of their job) internet users. None of the participants had viewed the homepages used in the study prior to their participation.

\subsection{Stimuli and Equipment}

Prior to the selection of the stimuli, a survey was conducted to identify the position of the 'About Us' link on homepages. Fifty European and fifty US E-Commerce sites were chosen on the basis of their sales. $80 \%$ of the homepages placed the 'About Us' link on the top of the page as a global navigation its position in the navigation bar varied on different websites. On the basis of this survey, we concluded that the convention is to place the 'About Us' link at the top of the page. This led to the assumption that Web users will be used to finding the 'About Us' link on the top of the page or at least expect to find it around that position based on their previous 
experiences. Therefore, the position of the target link in the repeated exposures session (exposures 2-7) was on the bottom of the page.

Ten UK E-Commerce homepages were selected as stimuli for this study: Cover4students www.cover4students.com (campus insurance), Hatton Garden Online www.hattongardenonline.com (jewellery), Diamond Daisy www.diamonddaisy.com (jewellery), Travelodge www.travelodge.co.uk (accommodation), Travel Bag www.travelbag.co.uk (travel), Train Line www.thetrainline.com (travel), Saga Holidays www.sagaholidays.com (holidays), Hotel net www.hotelnet.co.uk (accommodation), Past Times www.past-times.co.uk (gifts), To Book www.tobook.com (accommodation). The criteria for choosing homepages was that pages should have: i) a design layout that fits within the computer screen (17 inches flat screen with a resolution of 1024 by 768 pixels) without requiring scrolling; and ii) having both a top- and bottom-page navigation bar where the 'About Us' link could appear.

The pixel size of the target design element 'About Us' along with its presentation format (emboldening) and position within navigation bar slightly varied across homepages. For example, the Past Times homepage had the three navigation bars at the bottom of the page and the 'About Us' link appeared in the middle one.

Eye movements were recorded using an ASL (Applied Science Technologies) 504 eye tracking remote pan-tilt camera [1] capturing eye movement data at a sample rate of $60 \mathrm{~Hz}$. The presentation of the stimuli was controlled by means of the Gaze Tracker $^{\mathrm{TM}}$ software and presented on the screen viewed by participants from a distance of $55 \mathrm{~cm}$ from the screen. The Gaze Tracker ${ }^{\mathrm{TM}}$ software also records the eyemovements and enables viewing the data and output statistics based on researcherdefinable regions of interest on the web page (e.g. A-F in Figure 2).

\subsection{Procedure}

The duration of a session including the briefing and calibration process was approximately thirty minutes. The session started by the researcher giving an introduction of the eye tracking equipment and the study to the participant. The participant completed a consent form and a background questionnaire. The questionnaire captured age, gender, previous internet experience, and frequency and purpose of internet use. The participant was asked the following questions regarding the 'About Us' link: Where do you look when you want to find information regarding the company? Where do you prefer to find it? These questions were aimed to collect information about the user's expectations and preferences regarding the placement of the 'About Us' link before the eye-tracking session.

The researcher then calibrated the eye tracking camera for the participant. The participant was asked to look at each web page and find the 'About Us' link. The participant was asked to say aloud where on the interface they found the 'About Us' link in order to indicate that the task had been completed, so that the researcher could press the 'enter' key on the keyboard for the next page to appear. There was no time limit for the task so as to encourage a natural navigation of the web page. To avoid the researcher's reaction times influencing the data, the eye movement data were used as a measure of the task completion times. After the eye tracking session, the participants were asked: 'Where would you like to find the 'About Us' link? What do 
you think about the web pages you just saw? Was it easy to find the 'About Us' link'? These questions aimed to collect information about the user's perceptions and preferences regarding the 'About Us' link after the repeated exposures session.

\section{Results}

\subsection{Repeated Exposures Effect}

The study's first question aimed to examine whether users adapt their expectations of where to look when presented with repeated exposures of the 'About Us' link at the bottom of the page (an unconventional position). The qualitative data from the preand post-session questions along with eye tracking measures such as time to target fixation were analysed. The descriptive statistics of the scores to target fixation across repeated exposures are shown in Table 2. There is a difference between the sum of time to target fixation for the first of the repeated exposures (exposure 2) 179.10 and the last of the repeated exposures (exposure 7) 38.70 .

Table 2. Descriptive statistics of sums of time to target fixation for repeated exposures in milliseconds

\begin{tabular}{|l|l|l|l|l|r|l|}
\hline Exposures & $\mathbf{N}$ & Minimum & Maximum & Sum & Mean & $\begin{array}{l}\text { Std. } \\
\text { Deviation }\end{array}$ \\
\hline Exposure 2 & 10 & 3.52 & 38.86 & $\mathbf{1 7 9 . 1 0}$ & $\mathbf{1 7 . 9 0}$ & 11.41 \\
Exposure 3 & 10 & 1.44 & 44.97 & 120.16 & 12.01 & 13.11 \\
Exposure 4 & 10 & 0.72 & 17.98 & 64.63 & 6.46 & 5.65 \\
Exposure 5 & 10 & 1.53 & 31.64 & 83.26 & 8.32 & 9.99 \\
Exposure 6 & 10 & 1.20 & 41.13 & 122.62 & 12.26 & 14.09 \\
Exposure 7 & 10 & 0.91 & 6.80 & $\mathbf{3 8 . 7 0}$ & $\mathbf{3 . 8 6}$ & 1.61 \\
Exposure 8 & 10 & 2.53 & 16.16 & 58.48 & 5.84 & 3.99 \\
Exposure 9 & 10 & 1.80 & 22.86 & 91 & 9.09 & 6.79 \\
\hline
\end{tabular}

A non-parametric Trend test [28] was applied to test for a trend of learning where to look when presented with a sequence of six repeated exposures of homepages where the 'About Us' link appears on the bottom of the page.

A Page's L trend test [28] on the ranked scores of time to target fixation for the repeated exposures revealed a significant trend across exposures: $L(10,6)=792$, $\mathrm{p}<0.05$. A trend of time to target fixation decreases as the number of exposures increases is shown in Figure 1.

The eye tracking measures were complemented by the qualitative data as retrieved from the pre- and post-session questions. When the participants were asked before the eye tracking session where they expected to find the 'About Us' link they answered 'on top of the page' or it doesn't matter as long as they can see it. But, when the participants were asked after the eye tracking session where would they like to find the 'About Us' link they answered 'on the bottom of the page'. In addition to the eye tracking data where a trend of adaptation was found as exposures increased, the modification in answers from the pre- and post-session questions suggests an influence of change in preferences of where the 'About Us' link is expected to appear. 


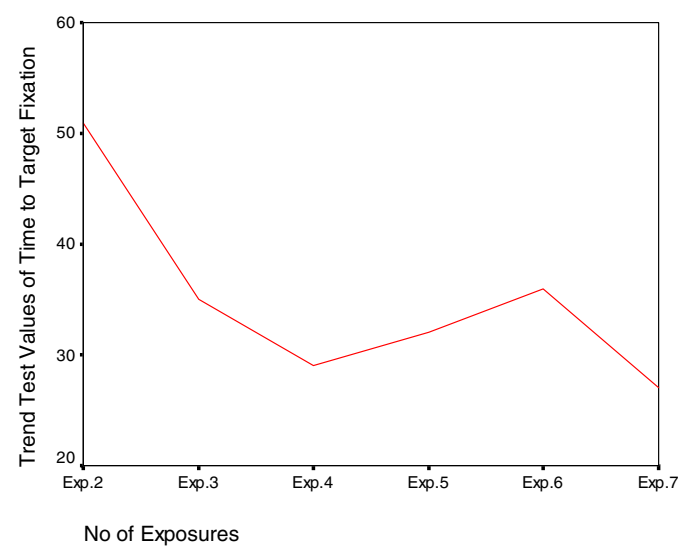

Fig. 1. Trend Test of time to target fixation across exposures

\subsection{Alternative Design Layout Effect}

The study's second question aimed to examine if users adapt quickly to alternative design layouts such as finding the 'About Us' link in an unconventional position.

A two-tailed paired t-test was used to establish whether there were significant differences between the time to target fixation before the repeated exposures (About Us link at the bottom of the page) and after repeated exposures (About Us link at top of the page) at the $5 \%$ alpha level of confidence. There is evidence that the users found the target link quicker when the 'About Us' link was placed at the top of the page even after having seen it on the bottom of the page in the repeated exposures session as $\mathrm{t}(9)=3.351, \mathrm{p}<.05$.

After transforming the raw scores using a two related samples Wilcoxon test, the distributions between the time to target fixation after the repeated exposures (About Us link at top of the page) and after the alternative exposure (About Us link at bottom of the page again) were compared at the 5\% alpha level of confidence. Despite the significance difference found when the About Us link is presented on the top of the page, when it is then presented again at the bottom of the page there is a significant difference as $\mathrm{t}(9)=1.98, \mathrm{p}<.05$.

The results indicate that when placing the 'About Us' link on the top of the page, users find it quicker than when placing it at the bottom of the page which might be the effect of their previous experiences as indicated in their self-reports and also as per the normal conventions of the About Us link's placement that we found in our survey of leading E-Commerce sites. But when presented with the 'About Us' link at the bottom of the page again after the repeated exposure a second expectation has been developed possibly caused by the consistent placement of the 'About Us' link on the bottom of the page over the repeated exposures.

\subsection{Before and After Repeated Exposures Effect}

Granka et al. [14] used a location grid to analyse eye movements in relation to position on the user interface. This enabled the classification of initial expectations 
and visual attraction to specific design elements. Similarly our study reported here used a location grid of six equal areas (see Figure 2) to determine the locations of 'initial gaze' and 'entry points'.

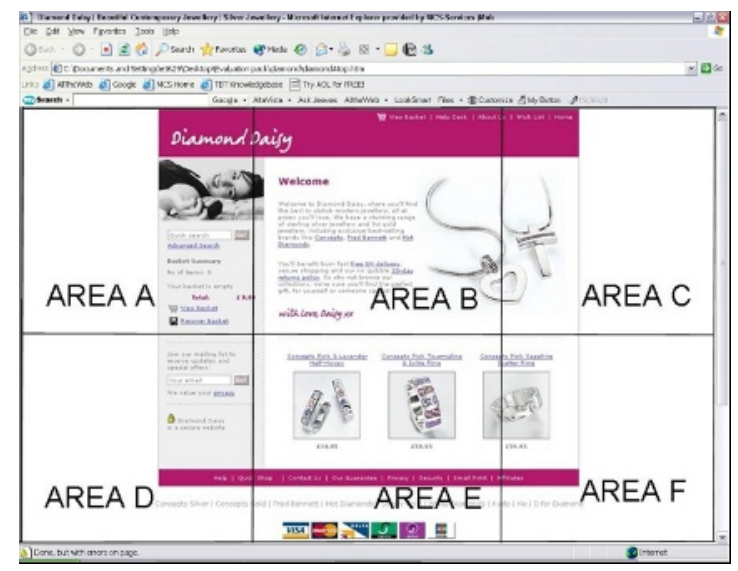

Fig. 2. Sample location grid to assess effect of design element location

The location of 'initial gaze' for each participant was measured during the first 50 milliseconds of the homepage appearing on the screen to determine where the participant expected to find the 'About Us' link. 'Initial gaze' for all participants was always in areas A or B (i.e. the top left or top middle of the screen) for both exposures 1 and 10. None of the 'initial gazes' focused on the right side or bottom of the screen (areas C, D, E, F in Figure 2). This is more of an indication of similar visual search strategies of initial gazes starting from the upper part of the page rather than any indication of user-adaptation across exposures. The very small amount of time (50 milliseconds) during which 'initial gazes' where measured might not have allowed the observation of any possible scan path modification. Therefore, the location of the 'entry point' for each participant was measured during the first 250 milliseconds of the homepage appearing on the screen to indicate where the participant first fixated. The 'entry points' were not consistent across participants and varied from homepage to homepage. Nevertheless none of the 'entry points' were in the right side of the screen (areas $\mathrm{C}$ and $\mathrm{F}$ in Figure 2). This might be influenced by the visual attraction of specific design elements rather than purely consistent placement of the 'About Us' link.

By comparing the users' scan paths in Exposure 1 and Exposure 10, five users where found to have modified their scan patterns from the first to the last exposure after being presented with the repeated exposures session. They started their scan paths in the upper area of the screen (areas A and B) whereas after the repeated exposures session they started their scan paths in the lower part of the screen (areas D and E) suggesting user-adaptation after finding the 'About Us' link at the bottom of the page. When looking at the profile of these five users we found that they were frequent internet users (regularly throughout the day) and used the internet as part of their everyday work activities suggesting that they were highly skilled internet users 
which might explain their quick adaptation to consistent placement of specific design elements such as the 'About Us' link. Despite a trend of user-adaptation during the repeated exposures session was found, the variance of scores of time to target fixation lead to the examination of the effect specific design characteristics of the target link.

\subsection{Effect of Design Characteristics}

Despite a trend of decrease in time to target fixation as exposures increased the variance of scores to target fixation lead to the examination of the effects of particular design characteristics of the target design element.

Table 3. Descriptive statistics of sums of time to target fixation for homepages regardless exposure in milliseconds

\begin{tabular}{|l|l|c|c|l|r|r|}
\hline \multicolumn{1}{|c|}{ Web Pages } & N & Minimum & Maximum & Sum & Mean & $\begin{array}{c}\text { Std. } \\
\text { Deviation }\end{array}$ \\
\hline Cover4students & 10 & 1.20 & 17.78 & 56.30 & 7.03 & 4.94 \\
Hatton & 10 & 1.88 & 32.80 & 99.19 & 12.39 & 12.00 \\
Past-times & 10 & 2.95 & 44.97 & $\mathbf{1 9 0 . 2 2}$ & 23.77 & 17.50 \\
To book & 10 & 2.23 & 22.86 & 56.45 & 7.05 & 7.25 \\
Travel bag & 10 & 1.53 & 13.94 & 46.77 & 5.84 & 4.15 \\
Diamond & 10 & 3.49 & 22.86 & 84.77 & 10.59 & 6.30 \\
Hotel net & 10 & 1.80 & 17.86 & 49.80 & 6.22 & 5.99 \\
Saga & 10 & 0.91 & 17.98 & 54.57 & 6.82 & 6.30 \\
Train line & 10 & 1.44 & 5.21 & $\mathbf{2 7 . 2 3}$ & 3.40 & 1.13 \\
Travelodge & 10 & 0.72 & 26.38 & 88.34 & 11.04 & 8.88 \\
& & & & & & \\
\hline
\end{tabular}

It is apparent that certain web pages required more time for the 'About Us' link to be identified. This was measured for each homepage, regardless of its place in the exposure order (Table 3). The Past Times homepage had the highest scores for the time to target fixation 190.22, whereas the Train line homepage 27.23 had the lowest scores for the time to target fixation.

When considering the possible effect of specific visual characteristics design elements, three characteristics were explored: a) pixel size, b) text appearing bold or not, and c) the position of the 'About Us' link within the navigation bar.

The pixel size of the target design element 'About Us' link was measured using JRuler Pro [19]. A relationship between time to target fixation and pixel size was found, but it was not significantly correlated $(r=-.189, \mathrm{p}>0.05)$. So, small size in pixels did not always lead to increased time to target fixation.

When the 'About Us' link did not appear in bold or distinctive colour (the formatting effects used in the example websites), the time to target fixation increased, implying that without distinctive formatting the target was more difficult to locate. The position of the 'About Us' link within the navigation bar did not appear to influence the time to target fixation. However, when more than one navigation bar appeared on the bottom of the screen, the time to target fixation increased. 


\section{Conclusions}

\subsection{User-Adaptation}

When placing the target link in a consistent position over a series of exposures, the results show that users adapt to consistent placement of the target link and improve their visual search performance. A trend was found of more exposures leading to decreased time to target fixation. Eye movement data was complemented by self reports of change in expectations and preferences of where the target link was expected to be found. The reported results comply with previous research as conducted by Ehret [5] suggesting that users learn the locations of design elements over series of repeated exposures. On the contrary, McCarthy et al. [25] had found no evidence that performance improves when the target link is placed in expected positions. They found that users adapt quickly to unexpected design layouts.

When placing the About Us link at the top of the page as per the norm on most websites as shown by our survey, the results indicate that users' visual search performance is quicker than when placing the target link at the bottom of the page. It is assumed that primacy effects of the users' exposure to web pages might have influenced their visual search performance whereas influence of the repeated exposures develops as a secondary effect which was found to improve visual performance but does not override the effect from previous experiences.

\subsection{Impact of Design Layout Characteristics}

Distinctive formatting of design elements (e.g. emboldening and position within navigation bar) also influences users' visual search behaviour and shortens time to target fixation. However, this influence is secondary to the learning effects: the learning effects associated with consistency of placement of the design element over repeated exposures were observed regardless of the complexity and visual characteristics of the design elements.

\subsection{Utility of Results for Designers and Researchers}

This study should encourage designers to aim for consistency not only within a website but also consistency across websites. However, just placing design elements in similar positions on the user interface within or across a website does not guarantee efficient visual search. The presentation format of the design element influences user visual search behaviour as well. The results of the reported study indicated that design elements such as the 'About Us' link are not only located more quickly when presented in a consistent position over exposures, but also when they are presented in a distinctive format. For researchers the study demonstrates the usefulness of applying eye tracking as a usability evaluation technique for user interface designs. Although the reported study is limited to visual search of homepages, the methodology can be applied to other web pages and to a wide range of tasks. In addition, it shows how eye movement data can be triangulated with data from other evaluation techniques, including qualitative elicitation, to enrich the study's outcomes. Eye tracking contributes to design knowledge and evaluation by providing detailed capture of user interaction behaviour. 


\section{Acknowledgments}

The research in this paper is being supported by an EPSRC Research Studentship. Our thanks to Dr. Alvaro Faria (Open University, UK), Professor Robert Jacob (Tufts University, US), Dr. Ewald Kaluscha (University of Klagenfurt, Austria), Dr. James A. Renshaw (Leeds Metropolitan University, UK) and, Mr. Jonathan P. SanDiego (Open University, UK) for their feedback and advice.

\section{References}

1. ASL (Applied Science Laboratories) Model 504. http://www.a-s-1.com/model504.htm last visited 29th April, 2005

2. Campbell, D. and Stanley, J. Experimental and Quasi- experimental designs for research on teaching. In Gage, N. (Eds.), Handbook of Research on teaching. Rand McNally Company, Chicago, (1963), 171-216

3. Cowen, L., Ball, L., Delin, J. An eye movement analysis of web page usability. In Proc. of the BCS-HCI, (2002), 317-335

4. Dix, A., Finlay, J., Abowd, G. D., Beale, R. Human -Computer Interaction. Third edition, Prentice Hall, England, (2003)

5. Ehret, B., D. Learning where to look: location learning in graphical user interfaces. In Proc. of CHI (2002), 211-218

6. Ellis, S., et al. Windows to the soul? What eye movements tell us about software usability? In Proc. of the Usability Professionals' Association Conference (1998), 151-178

7. Fitts, P. M. The Information capacity of the human motor system in controlling the amplitude of movement. In Journal of experimental psychology, 47, (1954), 381-391

8. Fitts, P. M., Jones, R. E., and Milton, J. 1. Eye movements of aircraft pilots during instrument landing approaches. Aeronautical Engineering Review (1950), 9 (2), 24-29

9. Goldberg, J. and Kotval, P.X. Computer interface evaluation using eye movements: methods and constructs. International Journal of Industrial Ergonomics 24 (1999), 631-645

10. Goldberg, J. and Kotval, P.X. Eye movements and interface component grouping: an evaluation method. In Proc. of the human and ergonomics society 42nd annual meeting, (1998), 486-490

11. Goldberg, J. H., Stimson, M. J, Lewenstein, M., Scott, N. and Wichansky, A. M. Blink response, visual attention, and the www: Eye tracking in web search tasks: design implications. In Proc. of ETRA (2002), 51-58

12. Goldberg, J. H. and Winchansky, A. M. Eye tracking in usability evaluation. The Mind's Eyes: Cognitive and Applied Aspects of Eye Movements. R. R. J. Hyona, and H. Deubel. Oxford, Elsevier (2003), 493-516

13. Granka, L., Joachims, T., and Gay, G. Eye-Tracking Analysis of User Behaviour in WWW Search. In Proc. SIGIR (2004), 478-479

14. Granka, L., Hembrooke, H. Gay., and Feusner, M. Correlates of Visual Salience and Disconnect. Unpublished research report, Cornell University Human-Computer Interaction Lab (2004) http://www.stanford.edu/ granka/research.html last accessed 29th April, 2005

15. Halverson, T., and Hornof, A. J. Link colours guide a search. In Proc. of CHI, ACM Press, (2004), 1367-1370

16. Hansen, D., W. Committing eye tracking. PhD. Thesis, IT University of Copenhagen (2003) 
17. Horowitz, T.S., and Wolfe, J.M. Visual Search Has No Memory. In Nature, 357, (1998) 575-577

18. Hornof, A. J. and Halverson, T. Cognitive strategies and eye movements for searching hierarchical computer displays. In Proc. CHI 2003, ACM Press, (2003), 249-256

19. JRuler Pro. http://www.spadixbd.com/jruler/ last accessed $2^{\text {nd }}$ August, 2004

20. Jacob, R., and Karn, K. S. Eye Tracking in Human-Computer Interaction and Usability Research. The Mind's Eyes: Cognitive and Applied Aspects of Eye Movements. R. R. J. Hyona, and H. Deube (eds.). Oxford, Elsevier (2003), 572-605

21. Jones, W. P. and Dumais, S. T. The spatial metaphor for user interfaces: experimental tests of reference by location versus name. ACM transactions on Office Information systems, 4, 1 (1986), 42-63.

22. Josephson, S. and Holmes, M.E. Visual attention to repeated internet images: testing the scan path theory on the World Wide Web. In Proc. of ETRA., ACM (2002), 43-51.

23. Just, M. A., Carpenter P. A. Eye Fixations and Cognitive Processes. Cognitive Psychology 8, 9 (1976), 441-480

24. Lewenstein, M., Edwards, G., Tatr, D., and De Vigal, A. The Stanford Poynter Project. (2002). Available at http://www.poynter.org/eyetrack2000/ last accessed $29^{\text {th }}$ April, 2005.

25. McCarthy, J. Sasse, M.A. \& Riegelsberger, J. Could I have the menu please? An eye tracking study of design conventions. In Proc. of BCS-HCI, (2003), 401- 414

26. Nielsen, J., Molich, R., Snyder, C. and Farrell, S. E-Commerce User Experience. Nielsen Norman Group, Fremont, CA (2001)

27. Outing, S., and Ruel, L. Eye Track III, The Poynter Institute available at http://www.poynterextra.org/eyetrack2004/ (2004), last accessed $29^{\text {th }}$ April, 2005

28. Page, E. B. Ordered hypotheses for multiple treatments: A significance test for linear ranks. Journal of the American Statistical Association, 58 (1963), 216-230

29. Pan, B., Hembrooke, H., Gay, G., Granka, L., Feusner, M., and Newman, J. The determinants of web page viewing behaviour: An eye tracking study. In S.N. Spencer (Ed.), Proc. of ETRA, ACM SIGGRAPH (2004), 147-154

30. Pirolli, P. and Card, S. Information foraging in information access environments. In Proc. of SIGCHI (1995), 51-58

31. Rayner, K. and Pollatsek, A. The Psychology of Reading. Lawrence Erlbaum Associates (1994)

32. Renshaw, J., A., Finlay. J. E., Tyfa D and Ward R D. Designing for visual influence: An eye tracking study of the usability of graphical management information. In Proc. of Interact (2003), 144-151 